\title{
A Hybrid Line Search Technique with Modified Goldstein and Wolfe Conditions
}

\author{
Sawsan S. Ismael \\ Department of Math / College of Education \\ University of Mosul
}

Received

16 / 02 / 2011
Accepted

10 / 07 / 2011

\begin{abstract}
الملخص
شروط Wolfe و Goldstein المطورة يمكن استخدامها لغرض دراسة خاصية التقارب

لخوارزميـة تكراريـة غير خطية، بهذه الشروط تمكنا من إيجاد خط بحث هجين ملائم لتحقيق

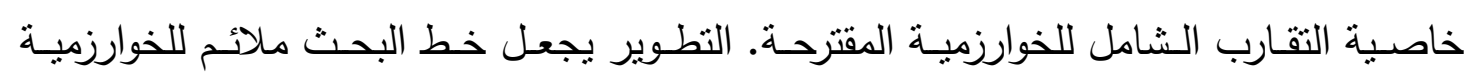

المقترحة. خط البحث المقترح تفوق على خط البحث الاعنيادي بأخذ تسع دوال لاخطية.
\end{abstract}

\begin{abstract}
Modified Goldstein or Wolfe conditions can be imposed on a hybrid line search to ensure the convergence property of an iterative nonlinear optimization algorithm to a stationary point. Modifying these conditions can make it significantly easier to find an acceptable step size. Our numerical results indicate that the new proposed line search beats the standard one ,for a selected nine test functions.

Keywords: Truncated Newton, Line-search (Modified) Wolfe conditions, (Modified) Goldstein conditions
\end{abstract}

\section{Introduction:}

Optimization might be defined as the science of determining the best solutions to certain mathematically defined problems, which are often models of physical reality, It involves the study of optimality criteria for problems, the determination of algorithms methods of solution, the study of the structure of such methods and computer experimentation with methods both under trial conditions and on real life problems. There is extremely diverse range of practical applications. The applicability of optimization methods is widespread, reaching into almost every activity in which numerical information is processed (science, engineering, mathematics, economics, commerce etc.) [3].

\subsection{The Goldstein Conditions:}


The Goldstein conditions for a step size $\alpha$ are:

$(G 1) f\left(x_{k}+\propto d_{k}\right)<f\left(x_{k}\right)+m_{1} \propto\left(d_{k} g_{k}\right)$

$(G 2) f\left(x_{k}+\propto d_{k}\right)>f\left(x_{k}\right)+m_{2} \propto\left(d_{k} g_{k}\right)$

Here $f$ is the function being minimized, $x_{k}$ is the current search position, $d_{k}$ is a search direction at $x_{k}, \propto$ is a step size found by the line search, $g_{k}=f^{\prime}\left(x_{k}\right),\left(d_{k}, g_{k}\right)$ denotes inner production and $m_{2}=1-m_{1}$ where $\left(d_{k} g_{k}\right)<0$ iff $d_{k}$ is a descent direction.

\subsection{The Wolfe Conditions:}

The Wolfe conditions may be used with the line search instead of the Goldstein conditions. The Wolfe conditions are:

$(W 1) f\left(x_{k}+\alpha d_{k}\right)<f\left(x_{k 6}\right)+m_{1} \alpha\left(d_{k} g_{k}\right)$

$\left(W_{2}\right) d_{k f} f^{\left(x_{k}+\alpha d_{k}\right)}>m_{2}\left(d_{k} g_{k}\right)$

As with the Goldstein conditions, $m_{1}, m_{2}$ are constants with $0<m_{1}<m_{2}<1$ and usually $m_{1}<0.5, m_{2}=1-m_{1}$

\section{Algorithms:}

\subsection{Algorithm (1) (Goldstein) [1]}

Let $\alpha$ be the initial step size (usually $\alpha=1$ ) and let $\mathrm{R}>1$ be a positive constant. Now perform $\beta:=\alpha$;

while not $\mathrm{G}_{1}(\alpha)$ do $\beta:=\alpha ; \alpha:=\beta / R$ enddo; while not $G_{1}(\beta)$ do $\alpha=\beta ; \beta ;=R * \alpha$ enddo

while not $G_{z}(\beta)$ do $\alpha: \beta=R_{s=}$ enddo;

The first iteration must terminate because $f$ is differentiate at $x_{k}$.

The second iteration must terminate because $f$ is bounded below.

Since if $G_{1}$ is false then $G_{2}$ is true.

We have the postcondition that $\alpha$ satisfies $G_{1}$ and $\beta$ satisfies $G_{2}$, and either $\beta=\alpha$ or $\beta=R \alpha$

Note that the two while loops can be placed in either order and that at most one of them will be performed.

\subsection{Algorithm (2) (Wolfe) [1]}

Let $\mathrm{R}>1$ and $0<\mathrm{r} \leq 0.5$ be positive constants.

Let $t$ be the initial step size (usually $t=1$ ) and perform

$\alpha=0 ; \beta:=\infty$;

while not $(\alpha=\beta)$ do

if $w_{1}(t)$ then $\alpha:=t$ else $\beta:=t$ end if;

if $w_{1}(t)$ and $w_{2}(t)$ then $\beta:=t$

else

choose a new $t$ with $\alpha<t<\beta$;

if $\beta=\infty$ then $t:=\max (t, R * \alpha)$

else $t:=\max ((1-r) * \alpha+r * \beta, \min (t, r * \alpha+(1-r) * \beta))$ 
end if

end if

end do

For the first iteration, $t$ is chosen to be initial step size. In later iterations, $t$ may be chosen by polynomial extrapolation or interpolation, but must be adjusted to ensure first that $\beta$ eventually becomes finite and subsequently that $\beta-\alpha$ decreases by a factor of $1-r$ in the worst case but even in the best case the factor is at least $r$, which can be unfortunate when $t$ is close to $\alpha$.

\section{Modified Algorithms:}

\subsection{Modified Goldstein:}

The two modified Goldstein conditions become as follows:

$\left(M G_{1}\right) f\left(x_{k}+\alpha d_{k}\right) \leq f\left(x_{k}\right)+m_{1} \alpha g T_{k} d k-m_{2} \alpha^{2} \mathbf{I} d_{k} \mathbf{I}^{2}$

$\left(M G_{2}\right) f\left(x_{k}+\alpha d_{k}\right)>f\left(x_{k}\right)+m_{2} \propto d_{k} g_{k}-m_{2} \alpha^{2} \mathbf{I} d_{k} \mathbf{I}^{2}$

where $m_{1}=0.0001$

and $m_{2}=0.9999$

$d_{k}$ is a search direction

$\alpha$ is a step size

$f$ is the function being minimized

$x_{k}$ is the current search position

$M G_{1}$ and $M G_{2}$ are modified forms of the standard Goldstein line search procedure with strictly descent property.

\subsection{Modified Wolfe:}

$\left(M w_{1}\right) f\left(x_{K}+\alpha d_{k}\right)<\left(x_{k}\right)+m_{1} \alpha d_{k}^{T} g k-m_{2} \alpha^{2} \mathbf{I} d_{k} \mathbf{I}^{2}$

$\left(M w_{2}\right) d k \cdot f^{\prime}\left(x_{k}+\alpha d_{k}\right)>m_{2} d g_{k} g_{k}-m_{2} \alpha^{2} \mathbf{I} d_{k} \mathbf{I}^{2}$

where $m_{1}=0.0001$

and $m_{2}=0.9999$

$d_{k}$ is a search direction

$\alpha$ is a step size

$g_{k}=f^{\prime}\left(x_{k}\right)$

$f$ is the function being minimized

$x_{k}$ is the current search position

$M w_{1}$ and $M w_{2}$ are modified forms of the standard Goldstein line

search procedure with strictly descent property.

\subsection{Theorem 1 (on modified Goldstein):}

Suppose that $f$ is bounded below and has Lipshitz continuous derivative $f^{\prime}$ on the basin $y: f(y) \leq f\left(x_{0}\right)$. Let $R>1$ be a positive constant. Let $d_{k}^{T} g_{k}<0$ and $\alpha_{k} \beta_{k}$ are chosen with $0<\beta_{k}<R \alpha_{k}$ and such that the step size $\alpha_{k}$ satisfies $\left(M G_{l}\right)$ and the step size $\beta_{k}$ satisfies $\left(M G_{2}\right)$ set $x_{k+1}=x_{k}+\alpha_{k} \dot{d}_{k}$ then either $\theta_{k}=0$ for some $k$ or else 
$\sum_{k=0}^{\infty}\left[\cos ^{2} \theta_{k}\left\|g_{k}\right\|^{2}\right]<\infty$

where $\theta_{k}$ is the angle between $d_{k}$ and $g_{k}$

Proof:

Let $m$ be $\min \left[m_{1}, 1-m_{3}\right]$ then by $(M G 2)$ we have

$f\left(x_{k}+\beta_{k} d_{k}\right)-f\left(x_{k}\right)>(1-m) \beta_{k} d_{k}^{T} g_{k}-m \beta_{k}^{2} \mathbf{I} d_{k} \mathbf{I}^{2}$

Hence by (MVT) we have for some c with $0<c<\beta_{k}$

$d_{k} \cdot f^{\prime \prime}\left(x_{k}+c d_{x}\right)>(1-m) d_{k}^{T} g_{k}-m \mid d_{k} \mathbf{I}^{2}$

$d_{k} \cdot f^{\prime}\left(x_{k}+c d_{x}\right)>d_{k}^{T} g_{k}-m d_{k}^{T} g_{k}-m \mathbf{I} d_{k} \mathbf{I}^{2}>d_{k}^{T} g_{k}-m\left(d_{k}^{T} g_{k}-\mathbf{I} d_{k} \mathbf{I}^{2}\right)$

so

$d_{k}\left[f^{\prime}\left(x_{k}+c d^{\prime} k\right)-f^{\prime}\left(x_{k}\right)\right]>\mathbf{I} d_{k} \| \mathbf{I} g_{k} \mathbf{|} \cos \theta-m\left(\mathbf{I} d_{k} \| \mathbf{I} g_{k} \mathbf{|} \cos \theta-\mathbf{I} d_{k} \mathbf{I}^{2}\right)$

Let $k$ be a Lipshitz constant for $f^{\prime}$ then

$\left\|f^{\prime}\left(x_{k}+c d_{k}\right)-f^{\prime}\left(x_{k}\right)\right\|<c_{k}\left\|d_{k}\right\|$ and so

$c_{k} \mathbf{I} d_{k} \mathbf{I}^{2} \mathbf{I} d_{k} \| \mathbf{I} g_{k} \mathbf{I} \cos \theta-m\left(\left\|d_{k}\right\|\left\|g_{k}\right\| \cos \theta-\left\|d_{k}\right\|^{2}\right)$

Also $c<\beta_{k}<R_{\alpha k}$

So

$\alpha_{1} k \mathbf{|} d_{1} k \mathbf{|}=-\left(m / R_{1} k\right) \mathbf{I} g_{1} k \mathbf{I} \cos \theta-\left(m / R_{1} k\right)\left(\mathbf{I} g_{4} k \mathbf{|} \cos \theta-\mathbf{|} d_{1} k \|\right.$

We note that both sides are positive, because $d_{k}$ is a descent direction so $\cos \theta_{k}<0$ from (MG1) we have

$f\left(x_{k}\right)-f\left(x_{k}+\alpha_{k} d_{k}\right)>-m \boldsymbol{\alpha}_{k} d_{k}^{T} g_{k}+m \alpha_{k}^{2} \mathbf{I} d_{k} \mathbf{I}^{2}>\left(\frac{m^{2}}{R_{k}}\right) \mathbf{I} g_{k} \mathbf{I}^{2} \cos ^{2} \theta_{k}>0$

So, the $f\left(x_{k}\right)$ are monotone decreasing and bounded below by $\mathrm{L}$ Hence,

$\left.f\left(x_{0}\right)-L \geq \sum_{k=0}^{\infty}\left[f\left(x_{k}\right)-f\left(x_{k}+1\right)\right)\right]>\left(m^{2} / R_{k}\right) \sum_{k=0}^{\infty}\left[\cos ^{2} \theta\left\|g_{k}\right\|^{2}\right]$

\subsection{Notes about Modified Wolfe Condition:}

\section{Corollary:}

Under the conditions of theorem 1 if we choose $\alpha_{k}, \beta_{k}$ to satisfy $M w 1, M w 2$ instead of $\mathrm{MG}_{1}, \mathrm{MG}_{2}$ respectively, then the same conclusion holds.

Proof: Let $m$ be $\min \left[m_{1}, 1-m_{2}\right]$ and proceed as in the proof of theorem, instead of applying the MVT to $M G_{2}$ use $M W_{2}$ directly to get

$d k_{k} f^{\prime}\left(x_{k}+b_{k} d_{k}\right)>(1-m) d_{k}^{T} g_{k}-(1-m) \| d_{k} \mathbf{I}^{2}$

Then proceed as before with $\beta_{k}$ in place of $\mathrm{c}$.

Note that although in practice $\beta_{k}$ is usually chosen so that $\beta k \geq \alpha k$ this condition is not required by either proof while the original corollary poured in [1].

\section{Modified Goldstein and Wolfe Line Searches:}

Use of the de-linked modified Goldstein conditions allows a very simple and quick line search to be implemented. 


\subsection{Algorithm (Modified Goldstein) :}

Let $\alpha$ be the initial step size (usually $\alpha=1$ ) and let $R>1$ be positive constant. Now:

$\beta=\alpha$;

while not $M G_{1}(\alpha)$ do $\beta:=\alpha ; \alpha:=\beta / R$ enddo

while not $M G_{2}(\beta)$ do $\alpha=\beta: \beta=R * \alpha$ enddo

The first iteration must terminate because $f$ is differential at $x_{k}$

The second iteration must terminate because $f$ is bounded below. Since if $M G_{1}$ is false then $M G_{\mathbf{z}}$ is true.

We have the post condition that $\alpha$ satisfies $M G_{1}$ and $\beta$ satisfies $M G_{z}$, and either $\beta=\alpha$ or $\beta=R \alpha$, we note that the two while loops can be placed in either order, and that at most one of them will ever be performed, while the original property found in [1].

\subsection{Algorithm (Modified Wolfe):}

Let $R>1$ and $0<\mathrm{r} \leq 0.5$ to positive constants.

Let $t$ be the initial stepsize (usually $t=1$ ) and perform

$\alpha=0 ; \beta:=\infty$

while not $(\alpha=\beta)$ do

if $\mathrm{MW}_{1}$ then $\propto:=t$ else $\beta:=t$ end if;

if $M W_{2}(t)$ and $W_{2}(t)$ then $\beta:=t$

else

choose a new $t$ with $\propto<t<\beta$;

if $\beta=\infty$ then $t:=\max (t, R=\alpha)$

else $t:=\max \left((1-r)^{* \alpha}+\mathrm{r}^{*} \beta, \min \left(\mathrm{t}, \mathrm{r}^{*} \alpha+(1-\mathrm{r}) * \beta\right)\right)$

end if

end if

end do

for the first iteration, $t$ is chosen to be the initial stepsize. In later iterations $t$ may be chosen by polynomial extrapolation or interpolation, but must be adjusted to ensure first that $\beta$ eventually becomes finite, and subsequently that $\beta-\alpha$ decreases geometrically with each iteration. The length $\beta-\alpha$ decreased by a factor of $1-r$ in the worst case, but even in the best case the fact is that least $r$, which can be unfortunate when $t$ is close to $\alpha$.

\section{Numerical Results}

To ensure the effectness of the two considered line search techniques we have to employ one of them namely [modified wolfe-line search] inanstandard CG-method (say FRCG method) and as follows:

\section{5-1 Algorithm (out lines)}

Step 1 :set $x_{1}, \epsilon, k=1, d_{1}=-g_{1}$ 
Step 2 :Find $x_{k-1}=x_{k}+\lambda_{k} d_{k+}$

Where $\lambda_{k}$ must be founded by algorithm (4-2)

Step 3 :Compute $d_{k+1}=-g_{k+1}+\beta_{k} d_{k}$

$$
\beta_{k}={ }^{\mathbf{|} g_{k+1} \mathbf{I}^{2}} / \mathbf{l} g_{k} \mathbf{I}^{2}
$$

Step 4 :If $k=n$ or Powell restarting condition satisfied go to step 1 else set $\mathrm{k}=\mathrm{k}+1$ go to step 2

Table (5-1)

Comparison between standard FR and wolf and the proposed modified method .

\begin{tabular}{|c|c|c|c|c|c|}
\hline \multirow{2}{*}{$\begin{array}{c}\text { Test } \\
\text { Function }\end{array}$} & \multirow{2}{*}{$\mathbf{N}$} & \multicolumn{2}{|c|}{ FR and wolf standard } & \multicolumn{2}{|c|}{ FR and wolf Modified } \\
\hline & & (Nof) & (NoI) & (Nof) & (NoI) \\
\hline \multirow{3}{*}{ Powll } & 100 & 256 & (126) & 245 & (114) \\
\hline & 500 & 1023 & $(505)$ & 1009 & (502) \\
\hline & 1000 & 2025 & (1005) & 2007 & (1001) \\
\hline \multirow{3}{*}{ Wood } & 100 & 828 & (276) & 872 & (323) \\
\hline & 500 & 3702 & (1007) & 3338 & (1000) \\
\hline & 1000 & 6797 & (1296) & 7739 & (2006) \\
\hline \multirow{3}{*}{ Rosen } & 100 & 296 & (103) & 296 & $(\mathbf{1 0 3})$ \\
\hline & 500 & 453 & (182) & 394 & $(152)$ \\
\hline & 1000 & 453 & (182) & 408 & (159) \\
\hline
\end{tabular}

In this comparison we have compared three well-known standard test function with high dimensions as asample for a comparison between the standard and modified line search criterion used in the standard FRCG method. Table (5-1) utilizes the numerical results for this comparisons, for nine case of (NoI) and (NoF) together, we have found that. The new proposed line search beats the standard one, in six cases out of nine while it has a comparable results in one of these cases.

The rate of improvement was about $34 \%$ in both (NoI) and $(\mathrm{NoF})$ according to our selected group of test Functions.

\section{Conclusions:}

This paper investigates an optimization algorithm with two modified line search ceiteria which are satisfy both modified Goldstein conditions $M G_{1}$ and $M G_{2}$ (or both modified Wolfe conditions $M w_{1}$ and $\left.M w_{2}\right)$

Although line search algorithms ensures that the step size is not too small or too long, this means that backtracking line search technique cannot be combined with any arbitrary search direction using algorithms (4-1) and (4-2). 


\section{Appendix}

1- Generalized Powell Function

$$
\begin{aligned}
F(x)=\sum_{i=2}^{n / 4}\left[\left(x_{4 i-3}-10 x_{4 i-2}\right)^{2}+5\left(x_{4 i-1}-x_{4 i}\right)^{2}+\left(x_{4 i-1}-2 x_{4 i-1}\right)^{2}+10\left(x_{4 i-3}-x_{4 i}\right)^{2}\right] \\
x_{0}=(\mathbf{3}, \mathbf{- 1}, \mathbf{0}, \mathbf{1}, \ldots \ldots)^{T}
\end{aligned}
$$

2- Generalized Wood Function

$$
\begin{aligned}
F(x)= & \sum_{i=2}^{n / 4}\left[100\left(x_{4 i-3}-10 x_{4 i-2}\right)^{2}+\left(1-x_{4 i-3}\right)^{2}+90\left(x_{4 i}-x^{2}{ }_{4 i-1}\right)^{4}+\left(1-x^{2}{ }_{4 i-1}\right)^{2}+1.0\right] \\
& x_{0}=(-\mathbf{3},-\mathbf{1},-\mathbf{3},-\mathbf{1}, \ldots \ldots)^{T}
\end{aligned}
$$

3- Generalized Rosenbrock Function

$$
\begin{gathered}
F(x)=\sum_{i=2}^{n / 4}\left[100\left(x_{2 i}-x^{2}{ }_{2 i-1}\right)^{2}+\left(1-x_{2 i-1}\right)^{2}\right] \\
x_{0}=(-\mathbf{1}, 2, \mathbf{1}, \ldots \ldots)^{T}
\end{gathered}
$$

\section{References:}

1) Bruce Christianson, Global convergence using de-linked Goldstein or Wolfe line search conditions, University of Hertfordshire, Hatfield A110 9AB, England.

2) L. Armijo, Minimization of functions having Lipschitz continuous first partial derivatives, Pacific Journal of Mathematics, 16(1), 1-3, 1966.

3) R. Fletcher, Practical methods of optimization. Second edition.

4) L.C.W. Dixon and R.C. Price, Numerical experience with the truncated Newton method for unconstrained optimization, Journal of Optimization Theory and Application, 56, 245-255, 1988.

5) A.A. Goldstein, Constructive Real Analysis, Harper and Row, London, 1967.

6) P. Wolfe, Convergence conditions for ascent method, SIAM Review, 17, 226-235, 1969 and 13, 185-188, 1971. 